\title{
Linderung des akuten Hustens durch Hustensaft mit Schleimhautschutz
}

\author{
Dr. Tobias Mück
}

Zwei randomisierte, verblindete, kontrollierte Multicenterstudien untersuchten den therapeutischen Effekt einer chemisch-mechanischen Barrierefunktion im Bereich der oberen Atemwege durch Hustensaft auf Basis von Polysaccharid-Harz-Honig bei akutem Erkältungshusten bei Kindern. Es zeigte sich eine signifikante Linderung des kindlichen Hustens durch den Schleimhautschutz im Vergleich zu Carbocystein-Sirup bzw. Placebo. Besonders scheinen Kinder mit starkem Husten und/oder nächtlichen Hustenepisoden zu profitieren.

$\mathrm{H}$ usten, und vor allem nächtlicher Husten, zählt zu den quälenden Begleiterscheinungen von Erkältungen. Zwei Studien veranschaulichen, wie es gelingt, über eine chemischmechanische Barriere die Schleimhaut zu schützen, deren Ausheilung zu fördern und damit den Husten zu lindern.

Die Hustensymptomatik bei einer akuten Erkältung wird wesentlich durch eine neuronale Hypersensitivität des Hustenreflexes ausgelöst. Rhinoviren, Bakterien oder Irritantien führen zu Entzündungsvorgängen an den Nervenendigungen der Mucosa und einer Neuromodulation der betroffenen Schleimhäute. Gelingt es, die Schleimhaut vor Noxen zu schützen, kann sie sich erholen und ausheilen. Geeignet hierfür sind mucoadhäsive Substanzen - wie pflanzliche Polysaccharide oder Honig - die sich wie ein schützender Film auf die Schleimhaut legen. Neben dem physikalischen Schutz wirken sie hydratisierend, sie verflüssigen den anhaftenden Schleim und machen ihn leichter abhustbar. Flavonoide binden als Antioxidantien freie Radikale, die infolge der Infektion im Körper entstehen können.

Eine im „World Journal of Pediatrics“ erschienene randomisierte, einfach blinde Multicenterstudie untersuchte die Wirkung eines schleimhautschützenden Hustensirups, der u.a. pflanzliche Polysaccharide, Honig und Flavonoide enthielt, auf Husten bei Kindern [1]. In die Studie eingeschlossen waren 150 Kinder im Alter zwischen 2 und 5 Jahren. Verglichen wurde der Sirup, ein Medizinprodukt mit Inhaltsstoffen auf natürlicher Basis, mit einem Carbocystein Sirup. Betrachtet wurde der Effekt auf Husten am Tag und in der Nacht als Begleiterscheinung einer akuten Infektion der oberen Atemwege. Besonderes Augenmerk lag auf der Besserung des nächtlichen Hustens. Dafür wurde die Nacht vor Beginn der Studienmedikation mit der Nacht nach Beginn der Studienmedikation verglichen. Bereits nach einem Tag Anwendung zeigten sich in Bezug auf alle untersuchten Parameter signifikante Unterschiede zwischen dem pflanzlichen Präparat und Carbocystein (siehe Abbildung). Ersteres senkte die Zahl der Hustenattacken etwa fünfmal besser als die Vergleichsmedikation, in Bezug auf die nächtlichen Hustenepisoden zeigte sich sogar eine zwölfmal bessere Wirkung des Verum-Sirups.

Eine im „Italian Journal of Pediatrics“ veröffentlichte randomisierte, multizentrische, doppelblinde und plazebokontrollierte Studie widmete sich ebenfalls der Untersuchung der Wirksamkeit eines Hustensirups mit natürlichen Molekülkomplexen [2]. Der Sirup, ein Medizinprodukt, enthielt pflanzliche Polysaccharide, Honig und Flavonoide. 102 Kinder mit Infekten der oberen Atemwege im Alter zwischen drei und sechs Jahren wurden in die Studie aufgenommen. Der Husten bestand seit mindestens sieben Tagen und höchstens drei Wochen. Untersucht wurde die Wirkung des Verum-Sirups im Vergleich zu einem Placebo-Sirup.

Kinder mit starkem Husten profitierten besonders von Einnahme des Verum-Sirups. In der Subgruppe von Kindern mit häufigem bis hin zu sehr häufigen und stark beeinträchtigenden Hustenepisoden besserte sich der Husten bis Tag vier nach Therapiebeginn bei 13 von 14 Kindern (93 $\%)$, im Plazebo-Arm dagegen bei 7 von 13 Kindern (54 \%) $(\mathrm{p}=0,03)$. Definiert war Besserung als keinerlei Husten bis

Evid Self Med 2021;1:210025 | https://doi.org/10.52778/efsm.21.0025

Afilliation/Korrespondenz: Dr. Tobias Mück, Consumer Healthcare Medical Affairs, Sanofi-Aventis Deutschland GmbH, Industriepark Hoechst, 65026 Frankfurt am Main, Germany (tobias.mueck@sanofi.com) 


\section{0,2}

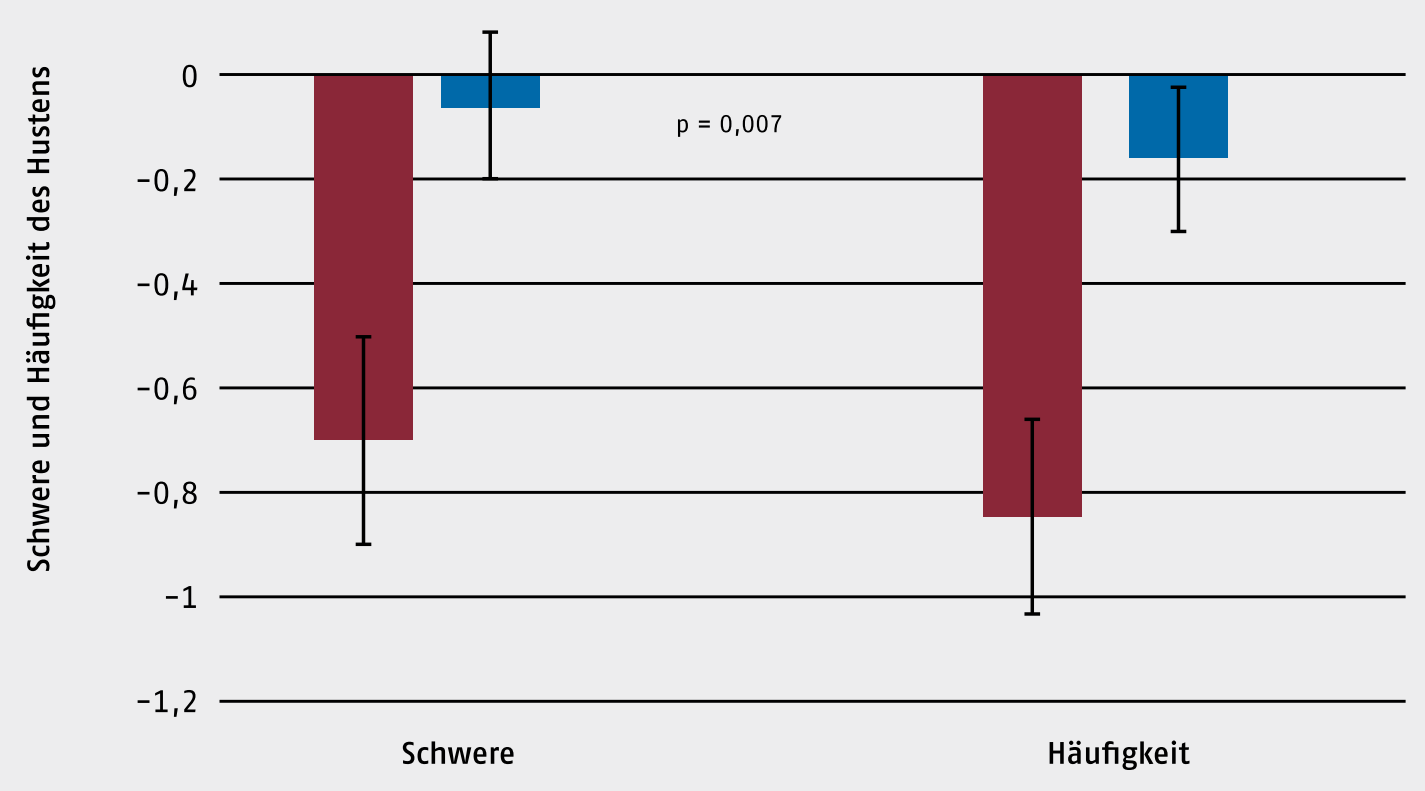

Abb.: Verringerung der Schwere und der Häufigkeit des Hustens nach 24 Stunden. Rot: Verum-Sirup (Hustensaft auf Basis von Polysaccharid-Harz-Honig) ( $n=75)$; Blau: Carbocystein-Sirup $(n=66)$.

maximal zwei kurze Hustenepisoden von etwa zehn Minuten pro Tag.

Zusammenfassung: Beide Studien veranschaulichen den positiven Effekt eines mechanischen Schleimhautschutzes auf die Linderung des kindlichen Erkältungshustens.

Besonders hervorzuheben ist die Besserung des nächtlichen Hustenreizes. Der schützende Film auf der Mucosa vermindert sowohl den Kontakt mit irritierenden Substanzen als auch mit Mikroorganismen und fördert damit die physiologische Regeneration. Die Anwendung ist sicher und effektiv, der Wirkeintritt ist bereits ab dem ersten Behandlungstag zu erwarten.

\section{Literatur}

Cohen HA et al. World J Pediatr. 2017;13:27-33.

Canciani M et al. Italian Journal of Pediatr. 2014;40:56.

Interessenkonflikt: Tobias Mück ist ein Angestellter von Sanofi.

Offenlegung: Medical Writing und Publikation finanziert von Sanofi Aventis Deutschland $\mathrm{GmbH}$.

\section{Informationen zum Manuskript}

Eingereicht am: 17.09.2020

Angenommen am: 26.12.2020

Veröffentlicht am: 16.08.2021 